\title{
Marge ou centre ? Où chercher la vérité ? L'orientalisme d'une Russe en Inde : Helena Petrovna Blavatsky
}

\section{Maya Burger}

\section{CpenEdition}

\section{Journals}

Édition électronique

URL : http://journals.openedition.org/edl/761

DOI : $10.4000 /$ edl. 761

ISSN : 2296-5084

Éditeur

Université de Lausanne

\section{Édition imprimée}

Date de publication : 15 septembre 2014

Pagination : 297-322

ISBN : 978-2-940331-35-2

ISSN : 0014-2026

\section{Référence électronique}

Maya Burger, «Marge ou centre ? Où chercher la vérité ? L'orientalisme d'une Russe en Inde : Helena Petrovna Blavatsky », Études de lettres [En ligne], 2-3 | 2014, mis en ligne le 15 septembre 2017, consulté le 18 décembre 2020. URL : http://journals.openedition.org/edl/761 ; DOI : https://doi.org/ 10.4000/edl.761 


\section{MARGE OU CENTRE? OÜ CHERCHER LA VÉRITÉ? L'ORIENTALISME D'UNE RUSSE EN INDE: HELENA PETROVNA BLAVATSKY}

Le Theosophist est un journal créé en Inde par la société théosophique en 1879. Il constitue la plateforme pour faire connaître les idées de la théosophie en Inde autant que dans le monde, notamment sous la plume de Mme Blavatsky qui en quelque sorte le dirige. Il est un lieu où se négocient et s'affrontent différentes formes de savoir dans le dernier quart du XIX ${ }^{\mathrm{e}}$ siècle. Blavatsky entend marginaliser le savoir d'une pensée occidentale matérialiste qu'elle décrit comme limitée ou étriquée par des religions et superstitions. Elle entend redonner une place (i.e. mettre au centre) à ce qu'elle appelle la sagesse universelle, dont l'Inde et le Tibet auraient préservé des dimensions perdues ailleurs. Dans ce contexte, les articles du journal révèlent toute une série de problèmes (traduction, interprétation) et de controverses qui témoignent des enjeux relatifs à qui est éligible de parler du savoir indien ou de "spatialiser» la sagesse. Ainsi la dispute avec le réformateur hindou Dayānanda Sarasvatī révèle des compréhensions divergentes des Védas. Bien que tentant de valoriser l'Inde et son héritage intellectuel, Mme Blavatsky perpétua la vision «romantisée» d'une Inde spirituelle, image qui conviendra bien aux formes de spiritualités pour lesquelles la théosophie a joué un rôle pionnier. Si elle a décentralisé l'Occident en l'invitant à changer de regard par rapport à l'Orient, elle a aussi marginalisé le savoir indien, notamment en le "théosophisant». Les premières années du Theosophist constituent également un matériel important pour analyser des perspectives indiennes de l'époque peu étudiées en raison de leur association avec un journal théosophiste relevant clairement de la marge.

\section{Introduction et problématique}

La théosophie, au sens de la société théosophique fondée en 1875, non seulement marque une étape dans l'histoire des religions en Occident, mais a aussi contribué à modifier l'histoire intellectuelle indienne dès la fin du XIX ${ }^{\mathrm{e}}$ siècle. En choisissant d'installer son quartier général en 
Inde, la société théosophique par ce geste a clairement manifesté l'importance qu'elle accordait à «l'Orient» et le rôle qu'elle entendait faire jouer aux acteurs orientaux dans la création d'un centre à partir duquel la «sagesse universelle» pourrait rayonner ${ }^{1}$. En découpant une page de l'histoire complexe des relations entre Orient et Occident, nous étudierons plus particulièrement les controverses liées aux sources des savoirs (sagesse, magie, révélation, ésotérisme, occultisme et sciences) et les stratégies de leur mise en place à la fin du XIX ${ }^{\mathrm{e}}$ siècle. Pour cette investigation, le centre d'attention est le journal The Theosophist, qui documente la diffusion de l'idéologie théosophique sur sol indien, mais également la diffusion des idées orientales dans le monde. Ce journal sert, par ailleurs, d'axe de réflexion pour parler d'un orientalisme indien.

L'orientalisme, dans le sens de connaissance orientale ${ }^{2}$, s'inscrit au cœur de ce débat autour des savoirs et peut être d'emblée défini comme une culture de la controverse, issue de la confrontation (encounter) entre des mondes d'ici et d'ailleurs, soulignant de manière remarquable que les lieux de la production du savoir sont déterminants. Dans ce débat, la théosophie blavatskienne occupe une place exemplaire et se distingue par des prises de position originales. De prime abord, tout pointe vers un orientalisme parfaitement marginal: une femme russe qui fonde une nouvelle ${ }^{3}$ religion, qui déplace le centre d'intérêt et d'établissement de sa nouvelle religion de l'occident vers l'orient, et comme si ce n'était pas déjà suffisant, en plein essor des sciences exactes, s'intéresse aux phénomènes occultes ${ }^{4}$. De surcroît, elle indique recevoir son savoir de maîtres

I. Blavatsky utilise le terme de religion de sagesse (H. P. Blavatsky, "What is Theosophy", The Theosophist, vol. 1, octobre 1879, p. 3: "Theosophy is, then, the archaic Wisdom-Religion, the esoteric doctrine once known in every ancient country having claims to civilisation", je souligne).

2. Au sens donc d'un orientalisme savant ne se réduisant pas aux productions occidentales et coloniales du savoir, et sans la coloration négative que prendra le terme dans la mouvance post-saïdienne.

3. Si j'emploie l'adjectif «nouvelle», ce n'est pas pour nier que Blavatsky emprunte son savoir à diverses traditions, mais pour souligner que sa systématisation du savoir universel et oriental est tout à fait originale.

4. Le terme "occulte» est entendu dans le sens que lui donne Blavatsky, qui veut rattacher son savoir occulte à la grande tradition savante d'un savoir caché à redéployer, se distanciant d'une acception du terme en tant que désignant des phénomènes mystérieux et inexplicables. Pour une clarification concernant l'histoire du terme, voir W. Hanegraaff, "Occult/Occultism». Pour le contexte plus général de l'occultisme 
transhimalayens certes invisibles, mais sources intarissables d'un savoir et d'une sagesse millénaires.

La théosophie est un mouvement élitiste et c'est également une élite de la société indienne qui est particulièrement concernée et dont il sera question ici. Pour Helena P. Blavatsky, la notion d'Orient équivaut à «berceau de sagesse" et elle considère l'orientaliste comme un savant occidental qui traduit le savoir oriental ${ }^{5}$. Néanmoins, on trouve chez elle des explications ou commentaires avant-coureurs qui auraient très bien pu émaner de Raymond Schwab ou d'Edward Saïd ${ }^{6}$. D'une part, elle voit la sagesse orientale comme nécessaire à l'avancement spirituel de l'Occident (si ce n'est à son avancement tout court): pas de progrès sans la philosophie orientale qu'il s'agit de faire renaître en Occident; et d'autre part, elle dénonce avec vigueur l'impérialisme occidental, les fausses lectures projetées sur l'Inde, telle que par exemple les chronologies historiques des orientalistes ${ }^{7}$, tout comme elle dénonce l'exclusion de l'opinion des Indiens eux-mêmes dans le débat ${ }^{8}$.

russe, mais dont il est difficile d'évaluer l'impact direct sur Mme Blavatsky, voir J. Mannherz, Modern Occultism in Late Imperial Russia; on y trouve également des positions critiques par rapport à la théosophie, par exemple autour de la question des lettres himalayennes (p. 158).

5. J'ai parcouru les écrits de Blavatsky durant sa période indienne, objet de la présente étude, et n'ai trouvé que ces emplois du terme, fort rares au demeurant. Elle emploie une fois l'expression de "système oriental" pour désigner la sagesse non pas disparue mais présente chez les initiés (cf. H. P. Blavatsky, Collected Writings, vol. 4, p. 493). Elle parle de "philosophie orientale» (cf. H. P. Blavatsky, Collected Writings, vol. 1, p. 270) pour inviter les occidentaux à reconnaître que le spiritualisme ne peut progresser sans la philosophie orientale (paru dans le Religio-Philosophical Journal, Chicago, 1877). J. A. Josephson, "God's Shadow», insère la pensée de Blavatsky dans les courants ésotériques autant que dans le cadre de l'histoire des religions naissante.

6. En faisant ici référence aux deux ouvrages majeurs pour l'histoire de l'orientalisme que sont La renaissance orientale et Orientalism.

7. Dans l'article "Antiquity of the Vedas", elle s'engage dans un débat visant à remettre en question les affirmations des orientalistes quant à la datation des Védas, valorisant également la perspective émique (The Theosophist, vol. 1, octobre 1879, p. $8 s q$ ).

8. "Western Science must inevitably be enriched by the contributions of the Indian, Sinhalese, and other mystics who will now find in the Theosophist a channel by which to reach European and American students of Occultism, such as never imagined, not to say seen, before.» (H. P. Blavatsky, "Antiquity of the Vedas», The Theosophist, vol. 1, octobre 1879 , p. 8). 
Le journal The Theosophist fut fondé à Bombay en 1879 par le tandem Blavatsky/Olcott, révélant à la fois les buts de Blavatsky, et les enjeux pour les hindous qui s'y sont exprimés. Ce journal est une parfaite illustration de la circulation des idées entre l'Inde et l'Occident, des controverses en jeu, ainsi que des questions de pouvoir liées à la détermination du savoir religieux. Le médium journalistique fait partie des nouvelles possibilités de répandre et négocier le savoir religieux, et Mme Blavatsky a su savamment en faire usage. Notons d'emblée que le Theosophist n'a pas fait l'objet d'une étude systématique 9 , alors que ce journal contient des articles innombrables d'Indiens de l'époque qui se sont exprimés sur leur tradition et son interprétation, et qui pourraient être une source de connaissance importante pour l'étude de l'histoire intellectuelle indienne de la deuxième moitié du XIX ${ }^{\mathrm{e}}$ siècle. Cet oubli ou amnésie fait elle-même partie de l'histoire de l'orientalisme, mais ce n'est pas pour autant que le journal occupe une place marginale dans cette histoire.

Mme Blavatsky réside durant 6 ans en Inde et fait partie des acteurs qui travaillent sur la profonde crise identitaire que les intellectuels hindous vivent autour des questions de rapport au pouvoir, d'emprise coloniale, de fractures identitaires, sociales et religieuses, tout comme des problèmes liés à l'entrée dans la modernité ${ }^{10}$. A l'aide d'exemples tirés $\mathrm{du}$ Theosophist, il s'agira pour nous d'une part de mettre en évidence les moyens par lesquels les protagonistes indiens et occidentaux ont donné une assise à leur savoir et d'autre part, de révéler le type de savoir traité et les sources employées à cette fin.

Après avoir brièvement présenté Mme Blavatsky et son séjour indien, puis le journal, deux controverses illustreront ensuite la manière dont le savoir «oriental» ${ }^{11}$ a pris sa place en Inde.

9. Même s'il existe ici ou là des commentaires sur des citations tirées du journal. A ma connaissance, il n'existe pas d'étude détaillée de ce journal dans la perspective d'une "entangled history».

IO. K. W. Jones, The New Cambridge History of India, vol. 3.1, notamment p. 167179 , résume la contribution de la théosophie au sein des mouvements de réformes qui bouleversent l'Inde britannique.

II. Par «savoir oriental», il faut entendre ici le savoir occidental sur l'Orient, auquel recourent tant les théosophes que les Indiens qui ont été formés aux perspectives orientales. Cf. supra, note 2. 


\section{Vie et perspectives de Mme Blavatsky (1831-1891)}

Dans le cadre d'une publication qui entend croiser les regards entre l'Inde et la Russie, il est tentant de s'intéresser à ce personnage russe haut en couleurs, qui a joué un rôle si important dans la question orientale. Véritable graphomane, Mme Helena Petrovna Blavatsky ${ }^{12}$ nous a légué plus de mille articles à côté des grands ouvrages qu’elle a rédigés, tels que Isis Dévoilée ou La Doctrine Secrète. Pour cette étude, je me concentre seulement et uniquement sur les années indiennes de Mme Blavatsky, c'est-à-dire de 1879 à 1885 , et ceci essentiellement autour des volumes du Theosophist publiés durant la même période ${ }^{13}$. Pourtant, il n'est pas facile d'aborder l'œuvre et la vie de Mme Blavatsky tant elle est une figure perçue de manière problématique, voire négative, et ceci fait aussi partie d'une réflexion sur la politique du savoir qui mérite un détour.

Helena Petrovna von Hahn est née en 1831 en Ukraine d'une très bonne famille. Elle épouse en 1849, à l'âge de 18 ans, Nikofor Blavatsky, le quittant après 3 mois. Commence alors une longue série de voyages qui selon ses propres dires, la mènent un peu partout dans le monde - fait très exceptionnel pour une jeune femme de l'époque. Cette culture du voyage formera une part importante de son bagage intellectuel sur le monde, complété par ses nombreuses lectures. Son premier voyage en Inde a lieu en 1852, à l'âge de 21 ans. Elle y retourne en 1855/56, puis en 1868, à 37 ans, pour enfin accéder au Tibet ${ }^{14}$, où elle aurait rencontré de

I2. Le fait que je me concentre ici sur H. P. Blavatsky n'implique pas que le Colonel Olcott n'ait pas joué un rôle déterminant dans la fondation autant que le développement de la société théosophique, notamment en Inde. La postérité a néanmoins surtout retenu le rôle de H. P. Blavatsky, véritable figure charismatique du mouvement. C'est aussi elle qui a principalement écrit dans le Theosophist.

13. La vaste littérature théosophique est magistralement ordonnée et largement accessible sur internet. Je résume ici les parties les plus importantes de sa vie pour notre propos, telles que consignées par ses biographes, tout en étant consciente que bien des épisodes de sa vie restent obscurs. Pour les années indiennes, on peut se référer à The Theosophical Movement, 1875-1950. Ce volume, composé par des éditeurs anonymes, contient également des explications relatives aux controverses des lettres et des mahätma. Pour la vie de Blavatksy: <http://blavatskyarchives.com/index.htm>. Le Blavatsky Study Center (même site) publie The Theosophical Movement, 1875-1925. A History and a Survey, 1925. Voir aussi J. Godwin, The Theosophical Enlightenment.

I4. Il est bien sûr peu probable qu'elle ait visité le Tibet, mais elle a certainement dû voyager dans les contreforts himalayens. L'affirmation de s'être rendu au Tibet n'est pas propre à Mme Blavatsky: d'autres chercheurs religieux, notamment indiens, ont dit 
grands maîtres, Koot Homi et Morya, qui l'instruiront dans la religionsagesse dont elle se fera la prophétesse. Dès 1870 , ces derniers lui enverront des lettres qui constitueront à la fois l'autorité sur laquelle elle fonde son savoir occulte, et la raison d'innombrables disputes et querelles auxquelles elle se verra exposée durant sa vie ${ }^{15}$.

Dès les années 1870, elle s'installe en Amérique et fonde à l'âge de 44 ans, en 1875, la Société théosophique avec le Colonel Olcott. Le but explicite de la société, selon ses statuts, est la fraternité universelle, l'encouragement de l'étude comparée des religions, philosophies et sciences, et l'étude des lois inexpliquées de la nature et des pouvoirs latents de «l'Homme».

S'inscrivant dans le sillage de l'héritage romantique, elle place l'Orient (et surtout l'Inde) au centre de sa réflexion, non pas comme un Orient mythique, mais bien comme lieu recelant un bien que l'Occident aurait perdu. C'est cette conviction qui la conduira à concrétiser avec le Colonel ce dont d'autres avaient seulement rêvé, s'installant durant 6 ans en Inde pour y établir de manière permanente le centre officiel de la théosophie ${ }^{16}$.

Elle se différenciera des précurseurs comme Herder ou Schlegel, en ne voyant pas l'Inde comme témoignant de l'enfance de l'Europe ${ }^{17}$, mais comme un interlocuteur atemporel, ou plutôt comme le siège, le centre, du savoir ésotérique ${ }^{18}$. Magie, ésotérisme, spiritisme ne sont

être allé au Tibet alors qu'ils n'avaient visité que les contreforts de l'Himalaya - ce qui contribue à confirmer l'aura dont le Tibet jouissait dans la quête du savoir spirituel (voir à ce propos $\mathrm{S}$. Thévoz, Un horizon infini).

I5. Il n'y a pas lieu de revenir ici sur ces querelles au sujet desquelles beaucoup a déjà été écrit. Pour un résumé rapide, on peut se référer à J. A. Santucci, auteur de la biographie de Blavatsky dans Dictionary of Gnosis \& Western Esotericism. La première lettre serait arrivée en 1870 dans la maison de la tante de Mme Blavatsky, en provenance de Koot Homi.

I6. Le quartier général de la théosophie fut d'abord situé à Bombay (Mumbai) puis, dès 1880 à Adyar (Chennai).

17. Ainsi Herder, dans sa quête de l'unité de l'espèce humaine, puis les romantiques, parleront de l'Inde ancienne comme de l'enfance de l'Europe, du berceau de l'humanité, de la spiritualité en son origine pure.

I8. La devise du mouvement est qu'il n'y a pas de religion plus élevée que la vérité; la théosophie est la base de toutes les religions. L'occultisme et la magie sont les faces pratiques de l'ancienne sagesse. Dayānanda Sarasvatī, dont il sera question plus loin dans ce texte, parle lui également de la vérité comme fondement ultime du savoir. Il nomme ainsi son ouvrage principal tout à fait à propos, Satyārtaprakāśa, Lumière sur la signification de la vérité. 
pas non plus de l'invention de Blavatsky, mais elle rassemble ces savoirs dans une théo-sophie dont elle postule un accès facilité dans la culture indienne, proche encore du savoir véritable ${ }^{19}$. S' installant à Bombay dès le 16 février 1879, elle fonde son journal The Theosophist en octobre de la même année. La société s'étend rapidement en Inde et le journal a bien sûr contribué à la faire connaître. Avec l'expansion et la renommée viennent aussi les critiques et la haine. En Inde comme ailleurs, elle exposera à maintes reprises ses talents de "magicienne", qui n'étaient cependant pas au goût de tous.

Sous le poids des critiques, attaques et controverses ${ }^{20}$, émanant autant des Occidentaux que des Indiens, Mme Blavatsky sera obligée de quitter l'Inde en 1885 (elle-même avance comme raisons la maladie et la déception qu'elle connaît avec les autres membres de la théosophie). Elle finira sa vie à Londres où elle meurt en 1891 après avoir fondé une loge en son nom, ainsi que le journal Lucifer qui se fait le porte-parole de son opposition au développement de la société occidentale et qui est, en quelque sorte, un pendant au Theosophist.

Retenons de cette vie extraordinaire que, bien que fondant son savoir sur des maîtres invisibles transhimalayens, elle s'impose elle-même comme une figure de maître vivante, détentrice d'un savoir nouveau et plus complet que ce que l'Occident avait à offrir. Les sources de son savoir émanent des voyages, des lectures et du contact avec le monde invisible. Elle le restitue sous forme de publications, de démonstrations et, une fois de plus, par des voyages ou déplacements visant l'enseignement. Notons d'une part la localisation physique du savoir (l'Orient-Tibet) qui dans sa perspective est un centre (et non une marge!), et d'autre part la figure du maître vivant, plaçant le savoir dans une lignée dont elle est l'initiée autorisée. Le modèle d'un savoir intérieur, augmenté par son universalisation à travers les voyages et les conférences, ne se laisse pas aisément exprimer par des critères de localisation, que ce soit le centre ou la marge.

19. La position de Mme Blavatsky à ce propos est décidemment ambiguë. Si elle plaide pour la réhabilitation de la sagesse orientale, sa position vis-à-vis de la modernité indienne est également marquée de la conviction que celle-ci reflète une dégénérescence d'un ancien savoir (voir aussi infra, note 28).

20. Tantôt perçue comme espionne, tantôt comme imposteur, les accusations à son égard n'ont pas manqué. Dans une longue lettre de 1885, elle explique les accusations subies et sa peine de quitter l'Inde dans ces conditions. Cf. H. P. Blavatsky, Collected Writings, vol. 1, p. 406-411. 
2. Le journal The Theosophist (1879-)

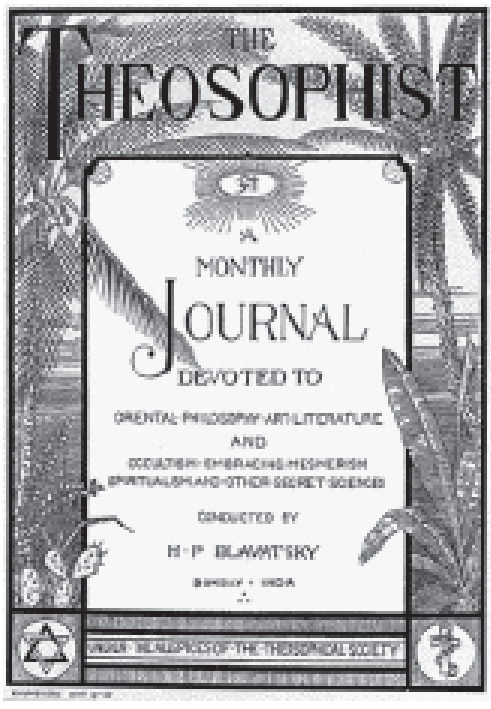

Ce journal, véritable collocation entre Orient et Occident, est l'œuvre conjointe de Olcott et Blavatsky, mais elle en est l'éditrice principale. Les deux protagonistes avaient un bon sens des affaires ${ }^{21}$ et il semble que le journal ait très vite connu le succès ${ }^{22}$. Vendu du nord au sud de l'Inde et majoritairement dans les villes, le journal doit peut-être son succès au fait que le couple a voyagé à travers l'Inde entière pour faire connaître ses activités.

Dès les origines du journal, Mme Blavatsky écrit plusieurs articles dans chaque volume. Dans «Namastae! [sic]», un article introductif au journal, elle explique la nécessité d'un organe par lequel les native scholars of the East puissent communiquer leur savoir au monde occidental, et «especially through which the sublimity of Aryan, Buddhistic, Parsi, and other religions might be expounded by their own priests or pandits, the only competent interpreters ${ }^{23}$.

Les éditoriaux sont riches en instructions relatives à la pensée et aux perspectives de Mme Blavatsky. Je me suis particulièrement intéressée à toutes les remarques et les notes de bas de page de la plume de Mme Blavatsky, qui, constituant une sorte de méta-texte, sont particulièrement illustratives de ses intentions.

Dans le premier numéro, elle explique ce qu'est la théosophie. A l'instar des idées en vogue dans la deuxième moitié du XIXe siècle, elle n'a rien à faire avec une révélation, mais répond à un esprit de

2I. Le journal devait être payé à l'avance par les abonnés afin d'en assurer la parution mensuelle. Le sens organisationnel de Olcott fut mis en évidence dans l'étude qui retrace son organisation du bouddhisme au Sri Lanka. Voir S. Prothero, The White Buddhist.

22. Selon J. A. Santucci, "Blavatsky, Helena Petrovna», p. 181, il y aurait eu une circulation initiale de 400 copies.

23. The Theosophist, vol. 1, octobre 1879, p. 1 . 
questionnement impartial et scientifique. Mme Blavatsky dit ne pas être concernée par la politique - l'homme intérieur n'étant guère concerné par ces balivernes. La fraternité universelle est aussi supérieure aux religions basées sur le dogmatisme et l'exclusion. Elle refuse toute forme d'anthropomorphisme du divin et se fonde sur l'autorité des maîtres anciens ${ }^{24}$.

Au moment de l'établissement de la société théosophique en Inde, la société indienne est dans une phase de remise en question importante ${ }^{25}$. Confrontée au regard britannique, exposée aux idées liées aux processus de modernisation plus généralement, ébranlée dans son identité et sa tradition, la société indienne vit durant le XIX ${ }^{\mathrm{e}}$ siècle des bouleversements à tous les niveaux. Bien avant l'arrivée des théosophes, les penseurs indiens expriment leur malaise vis-à-vis de la critique et de la dévalorisation de leur culture par le colonisateur ${ }^{26}$. Il en résulte des mouvements de réforme et de renaissance dont l'objectif est essentiellement de répondre au défi colonial, y compris celui posé par les missionnaires en termes de remise en question des traditions religieuses ${ }^{27}$. Il n'est donc pas étonnant que Mme Blavatsky rencontre un franc succès avec son Theosophist, dont

24. Cf. H. P. Blavatsky, "What is Theosophy», The Theosophist, vol. 1, octobre 1879 et également "What are the Theosophists", The Theosophist, vol. 1, octobre 1879. Dans les deux exposés, elle décrit l'héritage philosophique dans lequel elle s'inscrit, ainsi que le fondement unique et universel de toute religion que représente la théosophie. Santucci résume très bien son désir d'affiliation à l'ancien spiritualisme (et non au spiritualisme américain) : «[...] a system that should disclose [...] the "deepest depth" of the Divine Nature, and show [...] the real tie which binds all things together." (J. A. Santucci, "Blavatsky, Helena Petrovna», p. 179 sq.) et son ambition d'être rattachée à l'ancienne sagesse de l'ésotérisme occidental, augmenté de son fondement oriental.

25. Cet aspect a été particulièrement bien élaboré par W. Halbfass dans India and Europe, dans lequel l'auteur montre de manière précise l'échange d'idées entre les penseurs indiens et européens.

26. Ce sujet ayant fait l'objet de nombreuses études ces dernières années, je ne m'attarderai pas à relever et décrire l'ampleur du questionnement, ni à revenir sur les perspectives analytiques pour l'expliquer (et notamment tout le débat postcolonial et post-saïdien sur l'orientalisme). Pour un classique, voir D. Kopf, The Brahmo Samaj and the Shaping of Modern Indian Mind.

27. Plusieurs articles dans le Theosophist sont consacrés aux travaux missionnaires. A titre d'exemple, on peut citer celui d'A. Gordon ("Missions in India», The Theosophist, vol. 1, janvier 1880, p. 100 sq.), qui a fait l'objet d'une réponse par des missionnaires. Souvent le ton des articles est ironique (par exemple Melmoth the Wanderer, "Lo! the "Poor Missionary" ", The Theosophist, vol. 1, février 1880, p. 112 sq.). 
l'un des buts principaux consistait précisément à valoriser la tradition indienne. Elle fait très largement appel à l'idée de la grandeur passée de l'Inde, empruntant un discours tout à fait analogue à celui des orientalistes qui glorifiaient la seule Inde ancienne ${ }^{28}$. Elle s'en distancie cependant dans la mesure où il s'agissait aussi de restituer aux Indiens la fierté et la valeur qui avaient été ébranlées par le regard chrétien ${ }^{29}$. Un extrait du journal Patrika de Kolkata se fait l'écho de l'importance de cette valorisation, avant même le démarrage de la publication du Theosophist:

It will supply a long-felt national want - that of some organ through which native scholars could make themselves felt in the European and American worlds of thought. No Hindu need shrink from comparing the intellectual monuments left by his ancestors with those left by the progenitors of any Western people. The world has never produced but one Vedic philosophy, and the first to fathom the nature of the human soul were the Rishis. Since the THEOSOPHIST carefully abstains from politics, and its plan is one of a Universal Brotherhood, it should be welcomed by every sect and people throughout the world. And, as it recognizes the Aryans as the fathers of all religions and sciences, Hindus owe it their enthusiastic support ${ }^{30}$.

L'entrée dans le monde journalistique fut facilitée par l'éditeur du journal Pioneer, A. P. Sinnett ${ }^{31}$ à Allahabad, qui a joué un rôle déterminant

28. Rappelons que la glorification de l'Inde ancienne par les orientalistes, colons ou historiens des idées a eu un impact décisif sur l'autoperception des Indiens de leur tradition. Les orientalistes s'intéressaient généralement aux productions littéraires et religieuses en sanskrit classique (ou védique); pour les hindous des mouvements de réforme du XIX", les Védas et le "Védisme» sont l'autorité normative invoquée.

29. Mme Blavatsky parle toujours des Indiens en général. Le public visé du Theosophist était cependant un public éduqué, connaissant la langue anglaise. Certains Indiens en ont fait un reproche à la société théosophique, qui n'a pas pu ou voulu entrer en matière, car quelles langues choisir pour traduire les textes? Par ailleurs, Mme Blavatsky visait très clairement un public universel. Parfois, il est possible de se demander si l'audience principale n'était malgré tout pas le monde occidental, auquel elle aspirait à prouver le bien-fondé de sa vision.

30. The Amrita Bazar Patrika (Kolkata), 11 septembre 1879, cité dans "What the Press has said about the Theosophist», The Theosophist, vol. 1 (sans pagination). Malheureusement l'auteur n'est pas mentionné, mais il s'agit probablement d'un Indien.

3I. A. P. Sinnett (1840-1921), pour rappel, est celui qui a systématisé le contenu ésotérique des lettres des maîtres transhimalayens dans son ouvrage Esoteric Buddhism de 1883 . 
dans la crédibilisation de la théosophie aux yeux de la société indienne et plus généralement contribué à la faire connaître. Dans la perspective de l'attrait que Mme Blavatksy voulait exercer sur les "natives", comme elle dit, on peut citer un extrait de l'éditorial qui annonce la médaille qui sera décernée à celui, parmi les indigènes, qui écrira le mieux sur la gloire indienne:

To recognize that Aryavarta has a grand history and that the sons of the soil are her proper historiographers, and to stimulate a brotherly competition for a prize of real dignity, with ample guarantees for the impartiality of the awards, is to take a long step towards creating the feeling of nationality on which alone great states can rise ${ }^{32}$.

L'ambition politique de revaloriser le patrimoine indien apparaît clairement dans cette citation. De même, elle avait le souci de toucher les jeunes par son revival of Aryan wisdom, montrant clairement une volonté de restauration du savoir védique ${ }^{33}$.

Si nous regardons les trois premières années du journal, soit quelques 559 articles ${ }^{34}$, nous pouvons constater plusieurs éléments instructifs quant au profil que le journal veut d'emblée se donner. D’une part le choix des sujets est parlant, avec une majorité d'articles dévolus au religieux au sens large, à l'image de "The soul! Was the burning topic of debate» ${ }^{35}$. D'autre part la distribution des auteurs qui écrivent est

32. The Theosophist, vol. 1, p. 3 .

33. De fait, elle a eu quelques disciples ou co-théosophistes indiens très fidèles, mais elle a aussi eu des ennemis. Au moment de son départ en 1885, la société indienne a connu un déclin rapide avant l'arrivée d'Annie Besant comme nouvelle présidente en 1907. Sur le rôle transculturel d'Annie Besant, voir A. Malinar, "Western-Born But in Spirit Eastern..." ".

34. Sont ici comptabilisés les journaux publiés d'octobre 1879 à juin 1881. Il n'est pas aisé de compter de manière claire les articles, car il y a beaucoup de petits commentaires ou ajouts qui ne sont pas de réels articles, mais doivent être pris en compte dans notre perspective, car ce sont d'importantes sources pour connaître l'historique du journal ou les buts éditoriaux. Je remercie Nadia Cattoni pour la contribution à la systématisation des articles. An Index to the Theosophist est un autre outil qui permet de se faire une rapide idée des titres des articles du journal.

35. The Theosophist, vol. 1, p. 44. Le sujet le plus abordé est celui des religions institutionnelles (hindouisme, catholicisme, etc.) avec 87 articles suivis par 81 articles dévolus à la théosophie, 47 aux sciences occultes (magnétisme, etc.) ; 42 articles dédiés à la médecine, tant indienne qu'occidentale, 43 aux sciences indiennes (des mathématiques à la musique, yoga, etc.); 43 articles sont dédiés aux sciences dures (physique, 
significative: une majorité d'Indiens sont les auteurs d'articles (155) alors que 114 occidentaux ont pris la plume. 82 articles doivent être attribués à Mme Blavatsky, même si certains ne sont pas signés. Dans un contexte où la théosophie veut s'associer l'Inde comme partenaire à part égale, on ne peut que relever que le journal reflète très clairement cette prétention. Les thématiques abordées dans le journal montrent l'ambition de Mme Blavatsky de présenter un savoir exhaustif qui soit à même de parler de science autant que d'esprit, d'agriculture autant que d'archéologie. Il n'en reste pas moins que tout ce qui touche la science-sagesse est prépondérant dans ses analyses, et que l'Orient ne joue pas un rôle marginal dans le Thesophist, mais bien central - ce qui contribuera à nourrir des controverses à l'interne de la société. Ainsi, Anna Kingsford tenta de réorienter la société dans l'orbite chrétienne, exemplifiant l'enjeu du débat ${ }^{36}$.

Retenons que Blavatsky utilise le Theosophist autant pour faire connaître sa science occulte que pour faire internationalement connaître la grandeur de l'Inde et revaloriser ses habitants ${ }^{37}$. Elle travaille

agriculture, archéologie, etc.). Il existe toute une série d'articles sur des sujets de société, sur l'Inde, sur la mort, etc. difficiles à classer. Les biographies forment également un chapitre important ( 43 articles) dont la majorité sont faites par des Indiens sur des Indiens.

36. Très tôt dans la vie de la société, la controverse autour de la place de l'Orient est présente: George Wyld cherchait à rendre la société britannique fondée en 1878 indépendante de l'Orient (cf. J. A. Santucci, «Blavatsky, Helena Petrovna», p. 181 : "[...] more in step with the teachings of Christianity and less with the "atheism" of Blavatsky and anti-Christian views»), travail poursuivi par Anna Kingsford et Edward Maitland, qui créeront la Hermetic Society. Ajoutons en passant que ce débat relatif à la place de l'Orient n'est pas terminé aujourd'hui. Sur la notion d'orientalisme, son utilisation et les enjeux, voir M. Pasi, "Oriental Kabbalah and the Parting of East and West in the Early Theosophical Society".

37. Il est instructif de citer un extrait publié dans le journal anglais Public Opinion (novembre 1879) témoignant de l'impact qu'a eu la démarche sur ceux qui cherchaient à valoriser l'Orient. Ce texte est reproduit dans l'article «What the Press has said about the Theosophist", The Theosophist, vol. 1, juin 1880 (sans pagination): "There is a tone of elegance and scholarship about the whole of this periodical, which almost leads European readers to envy it. The translations of the Indian sacred documents given, have the advantage of being revised by Hindus, and there is, accordingly, a decidedly Oriental aspect to the whole work, which contrasts with the attempts certain German speculators have made to see the Vedas through the spectacles of Vaterland if not of Vater. [...] Still there is not a word in this paper which is offensive to any class of theologians. To show that it is a thoroughly learned production, it is merely necessary to 
clairement sur les deux fronts à la fois, indien et international, toujours dans le but d'une société universelle, sachant emprunter dans chaque cas les mots qui conviennent aux problèmes qu'elle a identifiés de manière claire. L'étude de deux controverses nous permettra d'en mesurer l'étendue.

\section{Deux controverses instructives tirées $d u$ Theosophist}

Deux controverses rapportées par le Theosophist illustrent les tensions autour de la «localisation" du savoir (marge? centre?) et autour des tentatives d'imposer une vérité à vocation universelle. Nous nous pencherons d'une part sur la controverse entre pouvoirs occultes versus savoirs religieux, entre Orient et Occident; d'autre part, nous évoquerons la tension entre tolérance et universalisme émanant de la dispute entre théosophie et Ārya Samāj, autour des interprétations du savoir védique données par Mme Blavatsky et par le réformateur indien Dayānanda Sarasvatī.

\subsection{L'occulte savoir mystérieux versus le religieux ou le savoir scientifique}

La théosophie se considère précisément comme une science de l'occulte, par quoi il convient d'entendre non pas une vague religion, révélation ou autre incongruité, mais bien une science rigoureuse étudiant les lois de l'invisible, à l'instar de la science qui étudie les lois du visible. Cette conviction ou perspective est régulièrement défendue par Blavatsky dans son journal, mais peut également être vue comme une stratégie pour accroître l'attractivité de son journal.

H. Blavatsky n'a pas seulement cherché à captiver l'attention des Indiens, mais voulait également toucher le plus large public possible, américain, européen (et probablement aussi asiatique ${ }^{38}$ ). Dans ce sens, elle reproduit dans le Theosophist (juin 1880) une lettre qu'elle reçoit d'un

indicate that the name appearing on the cover as conductor, is that of $\mathrm{H}$. P. Blavatsky, the erudite author of "Isis Unveiled", and one of the greatest living Orientalists. We wish that the THEOSOPHIST did not come out as far off as Bombay."

38. Elle mentionne que le journal est envoyé en Chine, au Japon et en Australie, outre l'Europe et les Etats-Unis, «Publishers Notice», The Theosophist, vol. 1, octobre 1879. 
éditeur américain auquel elle avait posé la question de savoir comment intéresser le public américain à son journal. La réponse est longue et très parlante du point de vue de l'histoire connectée autour de la question Orient-Occident. L'éditeur lui recommande de ne pas parler de religions asiatiques, mais d'illustrer et de confirmer par des preuves tangibles ce que l'on appelle «supernaturalisme»:

[...] Give the public interesting accounts of the marvels your Hindu pietist becomes capable of (according to tradition) when he attains the position of a Rishi or Arhat. Tell how this state is attained. Lift the veil from the psychological mysteries which are involved. Confute the pragmatical postulants of unconscious cerebration, hypnotism, and what not, as the causes and explanations of everything that puzzles them in Nature. Take, if you can, the jugglers of India as well as the Brahmans, describe their feats which have so bewildered the witnesses from the time of Kublai Khan until today. Give the world the first serious attempt it has seen to investigate the magic of India ${ }^{39}$.

Ce qui pourrait apparaître à première vue comme une lecture promulguant les mystères de l'Inde, est de fait une tentative d'approcher, à l'instar de Blavatsky, le monde du mystère scientifiquement. L'éditeur déclare que les religions asiatiques sont bien peu intéressantes, tout en reconnaissant qu'il y a des bribes de noblesse et de pureté, par exemple dans le bouddhisme. Mais ce qui l'intéresse et selon lui, intéresse le public occidental, c'est le savoir occulte. Ainsi conseille-t-il de parler des pouvoirs surnaturels et, les ayant soumis à des investigations sérieuses, de les expliquer de manière rigoureuse. L'éditeur met le doigt sur un problème qui divise les tenants du spiritisme et la société théosophique qui, sous la plume de Blavatsky, veut justement se distancier de l'improvisation mystérieuse en faveur d'une démarche rigoureuse.

Le nom de l'éditeur n'est pas cité: on ne mentionne que le fait qu'il est very clever. Que cette lettre soit ou non de la plume de Mme Blavatsky, il est clair qu'elle témoigne des stratégies adoptées par cette dernière. Ce qui devrait cependant retenir notre attention est le fait qu'elle cherche à s'assurer une clientèle américaine et que cette dernière serait friande

39. The Theosophist, vol. 1, juin 1880, "What the West Expects", p. 233 sq. 
de savoirs yoguiques ${ }^{40}$. Dans ce lien complexe, Orient-Occident, on ne peut que relever le caractère "prophétique» de cet énoncé. Quelques années plus tard, l'Indien Vivekānanda fera sienne cette injonction ${ }^{41}$ et, en tournant la mission dans l'autre direction, vendra le yoga comme le bien spirituel par excellence de l'Inde, mais un bien rigoureusement scientifique pour qui sait explorer les lois de l'invisible ${ }^{42}$.

L'exemple illustre, certes de manière implicite et à travers des stratégies de marché, la controverse entre spiritualité et science, et comment Blavatsky a réussi à fondre les deux dans sa science occulte capable de conquérir autant l'Orient que l'Occident. Le Theosophist est le lieu même où ce débat se cristallise répondant d'une part à la soif occidentale de soumettre tout savoir à des critères scientifiques, et d'autre part, à la conviction naissante de certains hindous, notamment des classes aisées et/ou lettrées, de détenir une sagesse ancienne à base scientifique.

\subsection{Interprétations contrastives des Védas: entre tolérance et universalisme}

Une femme russe et un moine brahmane vont convoquer tous les deux les Védas pour construire et imposer leur «religion». Ce qui débute par la quête partagée d'une fraternité universelle et la rencontre de deux esprits, se terminera dans une violente dispute ${ }^{43}$.

Dayānanda Sarasvatī (1824-1883), un brahmane dégoûté par les superstitions et idolâtries hindoues, fonda à Bombay en 1875 (la même année qu'est créée la société théosophique à New York) l'Ārya Samāj, la société des àrya, qui préconise un retour au Véda comme fondement du

40. Même si le terme n'est pas explicitement mentionné dans la lettre, notons que Mme Blavatsky reconnaît dès les premières pages du Theosophist qu'elle est une ferme adoratrice de Kapila, figure exceptionnelle pour elle des traditions indiennes, ainsi que de la maîtrise que les systèmes indiens offrent de l'esprit et du corps.

4I. On ne peut bien sûr pas l'affirmer avec certitude, mais il est possible que Vivekānanda, à l'affût des connaissances orientales, ait pris connaissance du journal et de son contenu. Lui-même préoccupé par la valorisation indienne, aura été attentif à une telle évaluation du marché potentiel.

42. Voir notamment son texte sur le rāja yoga, dans lequel il montre que le fondement de toute religion se trouve dans le Vedānta, qui se place en amont de toute manifestation religieuse particulière.

43. Un résumé de la rencontre se trouve chez I. Lubelsky, Celestial India, p. 97 et suivantes. 
seul savoir authentique. Ce svāmi, qui longtemps se promenait nu à travers l'Inde avant qu'on ne lui suggère qu'un habit de moine lui gagnerait de la crédibilité auprès des Occidentaux, rêvait de réinstaurer le savoir éternel et complet recelé par les textes originaux fondateurs de la civilisation aryenne, débarrassés de leurs scories tant musulmanes que chrétiennes et non frappés de l'état de dégénérescence illustré par des textes plus tardifs ${ }^{44}$. L'histoire de la rencontre entre Dayānanda et la théosophie commença bien avant la fondation des deux sociétés. C'est en 1870 qu'Olcott est informé, lors d'un voyage en bateau, de l'existence en Inde d'un mouvement destiné à réhabiliter les Védas comme l'unique source de sagesse et l'áryavarta, le pays qui la porte ${ }^{45}$. Dès 1875 , un échange épistolaire naît entre les deux mouvements dont les buts et stratégies semblent communs. Notons d'emblée que ces échanges passent par le filtre de la traduction, car le svāmi s'exprimait en hindi et sanskrit, langues auxquelles ni Olcott ni Blavatsky n'avaient accès, lui-même ne parlant pas anglais. Très certainement des questions de traduction seront en partie responsables des malentendus qui vont caractériser une relation turbulente entre les deux sociétés, mais qui n'éclateront que plus tard en dispute. Dans une lettre de 1878, Blavatsky se présente comme la porteparole de la vraie Inde, et elle mentionne Dayānanda comme le représentant de cette authenticité ${ }^{46}$. Les deux protagonistes vont tout d'abord

44. Dayānanda Sarasvatī et son mouvement ont fait l'objet d'études nombreuses et je me concentre ici sur la dispute avec la théosophie dont le Theosophist se fait l'écho.

45. En effet, Olcott rencontre sur un bateau entre les Etats-Unis et l'Angleterre en 1870 un certain Moolji Thackersey qui lui parle de Dayānanda et de la renaissance aryenne.

46. Voir, par exemple, "The Ârya Samâj. Alliance of Theosophy with the Vedic Society in the Far Orient", Collected Writings, vol. 1, p. 379-384. Parallèlement, Blavatsky publie dans un journal russe et parle précisément de ce grand yogi. Voir ainsi son texte paru dans le journal littéraire russe Russlij vestnik (Le messager russe), réédité en 1892 sous forme de livre: H. P. Blavatsky, From the Caves and Jungles of Hindostan, p. 18 et 20: "In short, all the attempts to re-establish the pure primitive monotheism of Aryan India have been a failure. They always got wrecked upon the double rock of Brahmanism and of prejudices centuries old. But lo! here appears unexpectedly the pandit Dayanand. None, even of the most beloved of his disciples, knows who he is and whence he comes. He openly confesses before the crowds that the name under which he is known is not his, but was given to him at the Yogi initiation. [...] During his five years of work Swami Dayanand made about two million proselytes, chiefly amongst the higher castes. Judging by appearances, they are all ready to sacrifice to him their lives and souls and even their earthly possessions, which are often more precious to 
se retrouver ${ }^{47}$ dans la restauration d'un passé glorieux, la réhabilitation d'un trésor perdu et la préoccupation avec le vrai savoir védique ${ }^{48}$. Elle l'invite dans le premier Theosophist à publier sa biographie inédite (en série), ce qui lui assura un succès auprès du lectorat indien. Elle-même utilise dès les débuts du Theosophist le terme d'ärya pour parler du bien proprement hindou, et d'äryavarta pour désigner le pays détenant cette sagesse, tout comme le réformateur indien.

La biographie traduite du svāmi fut annotée et commentée par Blavatsky, et un exemple significatif montre combien la question de

them than their lives. But Dayanand is a real Yogi, he never touches money, and despises pecuniary affairs. He contents himself with a few handfuls of rice per day. One is inclined to think that this wonderful Hindu bears a charmed life, so careless is he of rousing the worst human passions, which are so dangerous in India.»

47. Plusieurs extraits du programme de Dayānanda montrent la parenté avec la pensée de Blavatsky: sa vision d'une religion éternelle, universelle et seule vraie, contenue dans les Védas; la supériorité de la notion de vérité à toute forme de dogme; son opposition aux prêtres qui induisent les gens dans des fausses croyances. Enfin l'envie de créer une fraternité universelle: "In short I accept universal maxims: for example, speaking of truth is commended by all, and speaking of falsehood is condemned by all. I accept all such principles. I do not approve of the wrangling of the various religions, against one another for they have, by propagating their creeds, misled the people and turned them into one another's enemy. My purpose and aim is to help in putting an end to this mutual wrangling, to preach universal truth, to bring all men under one religion so that they may, by ceasing to hate each other and firmly loving each other, live in peace and work for their common welfare." (K. C. Yadav, The Autobiography of Dayanand Saraswati, p. 89). Voir également J. T. F. Jordens, Dayānanda Sarasvatī.

48. Dans une lettre de Dayānanda à Daya Ram en 1878, nous lisons: "The American Theosophical Society has become a branch of the Arya Samaj. They [Theosophists] accept the authority of the Vedas and are desirous to learn them.» (K. C. Yadav, The Autobiography of Dayanand Saraswati, p. 61). Il appelle Blavatsky tantôt Lady, tantôt Countess. Dans une autre lettre de 1879, nous lisons que Dayānanda autorise $\mathrm{H}$. S. Olcott à voter pour lui « upon all questions relating to the Theosophical Society which may be brought before the General Council for action in my absence» (K. C. Yadav, The Autobiography of Dayanand Saraswati, p. 63), et dans une lettre à Madho Dās: "Our union with these Sahibs will lead to the progress of the people of India." (K. C. Yadav, The Autobiography of Dayanand Saraswati, p. 64). Une lettre à Olcott dit: "You [Co. Olcott] have acted very wisely in negotiating with the Governor of Bombay. I am glad that the British Governement has no more suspicions regarding your stay and preaching in India the Vedic religion... I am glad to hear that you have started to reading Nagari. Your proposal of publishing a monthly journal is very sound. I only add a little to the name you have proposed: [...] call the journal The Theosophist or Arya Prakasha." (Juillet 1879, K. C. Yadav, The Autobiography of Dayanand Saraswati, p. 64). Il semblerait que le moine ait été entendu! 
la traduction a été déterminante dans cet échange. Blavatsky fait une remarque relative aux sages et anachorètes que Dayānanda aurait rencontrés dans l'Himalaya. Le terme est traduit par Mahatma (mahätma) et commenté par Mme Blavatsky comme référant à ces grands sages transhimalayens. Ce terme, à supposer qu'il l'ait bien employé ${ }^{49}$, ne devait certainement pas recouvrir cette vision chez Dayānanda qui, probablement, n'a jamais contrôlé les traductions et les notes. Cet élément n'est pas anodin dans la thèse de la diffusion d'un savoir authentique par la théosophie, car Dayānanda apporte la preuve vivante que l'on peut rencontrer les maittres dont Mme Blavatsky parle depuis longtemps. Loin d'être des figures imaginaires, il s'agit bien de sages itinérants tels que ceux que Dayānanda a rencontrés. Blavatsky définit les mahātma de Dayānanda (non nommés par lui) de la manière suivante:

The Mahatmas, or literally great souls, from two words - Mahâ, great and âtma, soul - are those mysterious adepts whom the popular fancy views as "magicians", and of who every child knows in India, but who are met with so rarely, especially in this age of degeneration. With the exception of some Swamis and ascetics of a perfectly holy life, they are few who know positively that they do exist, and are no myth, created by superstitious fancy. It will be given, perhaps, to Swami Dayananda, the great and holy man, to disabuse the skeptical minds of his degenerating countrymen, especially of this young decorated generation, the Jeunesse Dorée, [...] fed upon Western materialism, and inspired by the cold negation of the age, despise the traditions, as well as the religion of the forefathers, calling all that was held sacred by the latter, $\mathrm{a}$ « rotten superstition ${ }^{50}$.

Outre le fait de nous livrer un discours parfaitement orientaliste, Mme Blavatsky postule ainsi l'existence d'êtres de la qualité des "grandes âmes", les initiés spéciaux, avec lesquels elle est en contact. Elle explique également le terme de Yogi (dans le texte "Yog») utilisé par Dayānanda dans sa biographie en ces termes:

49. K. C. Yadav, The Autobiography of Dayanand Saraswati, a utilisé les textes hindi de la biographie, dont seuls les premiers épisodes sont accessibles en langue originale. Il ne mentionne pas ce terme. Il indique également qu'il a dû modifier la biographie telle qu'elle apparaît dans le Theosophist.

50. H. P. Blavatsky, "The Autobiography of Dayanund Saraswati, Swami», The Theosophist, vol. 1, décembre 1879, p. 67, note 4 (les accentuations sont dans l'original). 
A Yog: A religious «magician» practically. One who can embrace the past and the future in one present; a man who has reached the most perfect state of clairvoyance, and has a thorough knowledge of what is now known as mesmerism, and the occult properties of nature, which sciences help the student to perform the greatest phenomena; such phenomena must not be confounded with miracles, which are an absurdity ${ }^{51}$.

Là encore il apparaît clairement que H. P. Blavatsky sait tirer des parallèles avec la tradition occidentale, ici le mesmérisme, pour faire comprendre des phénomènes qu'elle postule comme d'égale qualité. Toutefois, me semble-t-il, les mahätma sont des initiés de plus grande valeur aux yeux de Blavatsky ${ }^{52}$.

L'idylle de la rencontre ne devait pourtant pas durer longtemps. Le Theosophist s'est fait le rapporteur de ces événements, ne laissant aucune chance au svāmi qui n'était pas aussi systématique dans l'archivage des échanges et promesses. En effet, Olcott était un archiviste méticuleux qui non seulement tenait un journal personnel, mais a aussi conservé et archivé des lettres et des informations relatives à l'Ārya Samāj. Dans leurs échanges épistolaires, il était prévu d'amalgamer les deux sociétés, dès que la théosophie s'établirait en Inde. Pour l'hindou en quête de la réhabilitation d'un honneur blessé, c'était une aubaine, tout autant que pour les théosophes qui y voyaient une insertion naturelle dans la société indienne. Cela ne sera cependant pas le cas, car Olcott remarque très vite que les intérêts n'étaient pas réellement partagés et plaidera pour laisser les deux sociétés indépendantes en en fondant une troisième appelée: Theosophical Society of the Arya Samaj of Aryavarta que les occidentaux théosophes pourraient décider de rejoindre ou non ${ }^{53}$.

La controverse s'est déclenchée autour de l'interprétation des Védas. Rappelons que Véda veut dire savoir, tout comme la théo-sophie se

5I. The Theosophist, vol. 1, octobre 1879, p. 12, note de bas de page (les accentuations sont dans le texte).

52. Je n'entre pas ici dans le débat sur la hiérarchie occulte qui, pour les théosophes, gouverne le monde (La Loge Blanche), ni sur la question de l'Agarttha, le centre du monde initiatique.

53. En mai 1878, les deux sociétés furent amalgamées, devenant The Theosophical Society of the Arya Samaj of Aryavarta (ou simplement Theosophical Society of the Arya Samaj, les deux noms s'écrivant sans signes diacritiques) qui n'a duré que jusqu'en mars 1882, date à laquelle Dayānanda exprime publiquement ses griefs contre les théosophes. 
veut savoir. Les théosophistes voyaient les Védas comme fondés sur une vérité impersonnelle et non localisée, alors que Dayānanda ${ }^{54}$ postulait un créateur et son pays, äryavarta ${ }^{55}$. Lorsqu'il découvre qu'au fond ses amis d'antan sont des athées, voire des bouddhistes, il les accuse ouvertement de fraude et de tromperie ${ }^{56}$. Dans un discours officiel de $1882^{57}$, Dayānanda énonce tous les griefs contre la société, ainsi que des réponses d'Olcott et Blavatsky qui essaient tous deux de rectifier ce qui a causé la colère de Dayānanda Sarasvatī. La controverse est ouvertement discutée dans le Theosophist, mais très nettement à l'avantage de la théosophie ${ }^{58}$.

54. Il est important de souligner que Dayānanda n'est qu'un exemple d'Indien qui parle dans le Theosophist et qui expose sa vision des Védas. Il conviendrait, bien sûr, de considérer également les contributions aux études védiques par les écrits d'autres auteurs indiens.

55. Dans son ouvrage majeur, Satyārthaprakāśa ("Lumière sur le sens de la vérité»), Dayānanda place le dialogue suivant, qui montre la terminologie qu'il utilise (p. 168): «ved me îśvar anek hai, is bāt ko tum mānte ho va nahīn? nahīn mānte, kyonki cāron vedon me esā kahīn nahīn likhā jisse anek î́var siddha ho. Kintu yah to likhā hai ki iśvar ek hai.» ("Dans le Véda, dieu est multiple, le sais-tu ou non? Tu ne peux pas le savoir, car dans les quatre Védas, il n'est nul part écrit que dieu soit multiple. Cependant, il est écrit que dieu est un.»). Ísvar est ainsi pour lui le dieu unique des Védas.

56. Mme Blavatsky voit le bouddhisme comme la pure religion védique, originaire du pays avant les transformations brahmaniques (The Theosophist, vol. 1, octobre 1879). De fait, Olcott et Blavatsky reçurent l'initiation au bouddhisme lors de leur voyage au Sri Lanka en 1880.

57. Déjà dans une lettre à Blavatsky en novembre 1880, il lui écrit: "You had come here to become disciples, now you wish to become teachers...» (K. C. Yadav, The Autobiography of Dayanand Saraswati, p. 68). En 1881, il écrit à Blavatsky: "I preach Vedic truths. I am thus a preacher and do not wish to be any more than this. You [Theosophists] call me some time member, some time something else. I don't wish to be honoured or to have any high position [in any organization]. What I really wish is a great thing [reform of India]. Hopefully, I shall succeed in getting this done by the help of God and cooperation and kindness of scholars and good men.» (K. C. Yadav, The Autobiography of Dayanand Saraswati, p. 69). En mars 1882: "It has been admitted that the Theosophist Society and its dictates are not worth considering. Therefore, all should remain away from it." (K. C. Yadav, The Autobiography of Dayanand Saraswati, p. $71 s q$.$) .$

58. Pour elle, cela apparaissait comme un échec explicable à partir de l'envie de Dayānanda que le samāj soit l'unique mouvement védique. «Alas! all this was written some time ago. Since then Swami Dayanand's countenance has changed completely toward us. He is, now, an enemy of the Theosophical Society and its two foundersColonel Olcott and the author of these letters. It appeared that, on entering into an offensive and defensive alliance with the Society, Dayanand nourished the hope that all 
L'universalisme et son apparente tolérance sont le fruit d'une vie de voyages pour Blavatsky qui a construit son savoir bribes par bribes, alors que Dayānanda n'avait pas quitté l'Inde et voyait ce pays, āryavarta, comme le centre de l'univers et les Védas comme le fondement complet et immuable de cette assertion. La fraternité universelle fait partie du programme de la société théosophique dès son départ, alors que Dayānanda reste profondément ancré dans le contexte de son pays, y compris pour ce qui concerne la suprématie brahmanique. Dayānanda voulait remettre l'Inde au centre du monde, alors que Blavatsky voulait offrir au monde la sagesse atemporelle, universelle et holiste ${ }^{59}$. L'origine brahmanique de Dayānanda ne lui permettait pas de tolérer la liberté avec laquelle Blavatsky utilisait ce que l'Inde avait de plus précieux et il ne ménage pas ses mots pour la dénoncer. Dans cette lutte entre deux partis convaincus d'avoir raison, Dayānanda ne pouvait supporter l'idée que les deux protagonistes parlaient du Véda sans connaître le sanskrit ${ }^{60}$.

Nos deux fondateurs de nouvelle religion s'accordent cependant sur la conception du rôle attribué au maître vivant, tant l'une que l'autre désirant incarner l'autorité du savoir qu'ils propageaient.

Cet exemple montre clairement que les attentes et l'interprétation des Védas du côté indien, via l'Ārya Sāmaj, et du côté américano-russe, via la théosophie, étaient complètement différentes. Il s'agissait pour l'un de la vérité ultime ${ }^{61}$ et pour les autres, de la base d'une spiritualité à

its members, Christians, Brahmans and Buddhists, would acknowledge His supremacy, and become members of the Arya Samaj. [...] Needless to say, this was impossible. The Theosophical Society rests on the principle of complete non-interference with the religious beliefs of its members."

59. Ce sont ses propres termes dans les pages introductives du Theosophist.

6o. Cela témoigne bien du fait que le «savoir oriental» se négocie autour des questions de pouvoir, d'authenticité et d'accessibilité. Rappelons à ce propos «[that] the champions of scientific orientalism in Europe suffered the same fate at the hands of the theosophists that Brahmanic tradition did at the hands of the former. They did not hesitate to appropriate anything that could serve their purpose, especially the most ancient references. They did not hesitate to either belittle, marginalise and sometimes even remove explicit references to the intermediary process of transmission and exegesis of texts without which they would not have access to them. For Max Müller as well as for the theosophists and most of the erudites of the time, it was western libraries that provided privileged access to knowledge." (D. Vidal, "Max Müller and the Theosophists", p. 21).

6I. Son ouvrage principal, Satyārthaprakāśa pose le fondement de son idéologie védique pour l'homme moderne. 
portée universelle ${ }^{62}$. Les deux avaient cependant un agenda pratique en commun, visant à revaloriser une identité hindoue.

L'Orient pour Blavatsky est un lieu et en même temps une universalité. Pour Dayānanda, il n'y a pas d'Orient et il n'y a pas de marge. Il y a le centre du monde, qui est les Védas et l'äryavarta.

\section{Conclusion}

La société théosophique a joué le rôle d'un centre de l'orientalisme en diffusant des connaissances orientales pour un Occident considéré comme marginal afin de comprendre l'universalisme d'une science occulte, fondée sur les lois de l'invisible. Le Thesophist a construit ce lieu de négociations des savoirs ${ }^{63}$ et constitue une illustration parfaite de l'histoire connectée des connaissances et compréhensions entre l'Inde et l'Occident. L'axe de diffusion du Theosophist incluait également la Russie, car Mme Blavatsky a publié dans tous les numéros des récits de Russes. De même, elle a publié (avec pseudonyme) ses années indiennes sous forme de feuilleton dans Russkij vestnik (Le messager russe) entre 1879 et 1882.

Le Theosophist a contribué à faire connaître certains aspects de l'Inde, de manière complémentaire à l'orientalisme savant et l'imaginaire populaire et exotique, laissant largement la parole aux Indiens. Ce n'était pas une affaire négligeable, puisque le journal fut largement diffusé aussi en Occident. Même si son étude, dans la construction occidentale du savoir, peut être qualifiée de marginale en raison de son association avec la théosophie et Blavatsky, il n'en reste pas moins que pour l'histoire de l'orientalisme, il constitue un apport central. Le journal non seulement reflète

62. La controverse pose bien sûr la question de «l'authenticité» d'une religion que les deux protagonistes cherchent à prouver, par rapport à celle qui serait fausse, comme l'a relevé P. van der Veer, Imperial Encounters. Cet élément est d'autant plus problématique que tant Mme Blavatsky que Dayānanda occupaient des positions fragiles dans leurs contextes "religieux» et épistémiques respectifs. Les interprétations et modifications proposées par Dayānanda n'étaient pas vues d'un œil favorable par l'orthodoxie brahmanique.

63. Il ne s'agit pas d'évaluer de manière critique le travail de Mme Blavatsky ou de la société théosophique, mais de montrer le rôle tout à fait exceptionnel joué par les deux dans l'histoire des savoirs orientaux, de l'occultisme et de la diffusion de l'orientalisme en Inde. 
l'orientalisme occidental, mais également l'orientalisme indien, fruit d'une relecture des traditions à la lumière des remises en questions du XIX $\mathrm{X}^{\mathrm{e}}$ siècle.

Derrière l'apparente volonté de fonder une fraternité universelle, et je crois qu'on peut sans autre dire que cette volonté de Mme Blavatsky était sincère, se profile cependant un couple de génie qui connaissait les recettes du succès et dont la volonté civilisatrice ne différait pas fondamentalement de celle des missionnaires chrétiens, tout en mettant l'accent sur ce qui leur apparaissait comme la vérité ultime. Dénonçant les méfaits des colonisateurs, tout comme la "stupidité» des missionnaires, le programme de Blavatsky opérait cependant sur un modèle analogue!

Peut-on alors parler d'une forme marginale d'orientalisme à l'intérieur d'un orientalisme plus général? Cela dépend à qui l'on s'adresse. Ayant emprunté à tous les niveaux des chemins de traverse, au sens propre de raccourci (amalgamant toutes les traditions) et au sens plus figuré de chemin peu "orthodoxe", la théosophie de Mme Blavatsky s'est vue très vite marginalisée, en dépit de son universalisme. C'est en fait surtout la fondatrice russe qui a suscité (et continue à susciter) une certaine gêne et a été reléguée dans les marges, alors même qu'elle représente le centre charismatique du mouvement. Son intention de réhabiliter l'Inde, lieu même de la science la plus complète, s'est soldée par la perpétuation de l'image d'un pays de l'occulte mystère. Cette ouverture orientale sur les faces cachées du monde s'est largement déversée dans toutes les néospiritualités globalisées du $\mathrm{XX}^{\mathrm{e}}$ siècle. A ce niveau alors, pas de doute, on passe des marges au centre! Si Blavatsky a contribué à décentraliser l'Occident, elle a aussi contribué à marginaliser le savoir indien en le théosophisant.

Lorientalisme des marges est un outil conceptuel pour articuler et réfléchir sur les liens interculturels. Il n'en reste pas moins que la spatialisation du savoir reste problématique, tant elle dépend des points de vue. Est-ce que l'universalisme peut avoir un centre? Est-ce que la rencontre peut en avoir un? La quête de la vérité, exercée par nos protagonistes ne peut pas être localisée, ni au centre ni dans la marge. Mais l'histoire de la quête du savoir "vrai" s'étudie en se focalisant sur les rencontres, les conflits, les regards croisés et enchevêtrés. L'histoire de l'orientalisme ne se laisse pas "universaliser", mais elle est indispensable pour situer nos méthodes dans l'approche de l'Orient (l'orientalisme compris).

Maya BURGer Université de Lausanne 


\section{BIBLIOGRAPHIE}

Blavatsky, Helena Petrovna, From the Caves and Jungles of Hindostan, London, Theosophical Pub. Society, 1908, online: <https:// openlibrary.org/books/OL14008813M/From_the_caves_and_ jungles_of_Hindostan>.

-, Collected Writings, volume I-IV, Adyar Madras/Wheaton, The Theosophical Publishing House/The Theosophical Press, 19661969.

-, Collected Writings, volume V-VI, Los Angeles, Blavatsky Writings Publication Fund, 1950, 1954.

Dayānanda Sarasvatī, Satyārthaprakaśa, New Delhi, Sārvadeśik ārya pratinidhi sabhā, 2002, repr. de l'édition de 1883 [1875].

Godwin, Jocelyn, The Theosophical Enlightenment, Suny Series in Western Esoteric Traditions, Albany, Suny, 1994.

Halbfass, Wilhelm, India and Europe: An Essay in Understanding, Albany, Suny, 1988.

Hanegraaff, Wouter (ed.), Dictionary of Gnosis \& Western Esotericism, Leiden, Brill, 2006.

—, "Occult/Occultism», in Dictionary of Gnosis \& Western Esotericism, ed. by W. Hanegraaff, Leiden, Brill, 2006, p. 884-889.

Jones, Kenneth W., The New Cambridge History of India, vol. 3.1: Socio-religious Reform Movements in British India, Cambridge, Cambridge University Press, 1989.

Jordens, J. T. F., Dayānanda Sarasvatī: His Life and Ideas, Oxford, Oxford University Press, 1978.

Josephson, Jason A., "God's Shadow: Occluded Possibilities in the Genealogy of "Religion”", History of Religions, 52/4 (2013), p. 309-339.

Kopf, David, The Brahmo Samaj and the Shaping of Modern Indian Mind, Princeton, Princeton University Press, 1979.

Lubelsky, Isaac, Celestial India: Madame Blavatsky and the Birth of Indian Nationalism, Sheffield, Equinox, 2012.

Malinar, Angelika, "Western-Born But in Spirit Eastern..." Annie Besant Between Colonial and Spiritual Realms", Etudes Asiatiques, 67/4 (2013), p. 1115-1154. 
Mannherz, Julia, Modern Occultism in Late Imperial Russia, DeKalb, NIU Press, 2012.

PAsI, Marco, "Oriental Kabbalah and the Parting of East and West in the Early Theosophical Society", in Kabbalah and Modernity: Interpretation, Transformations, Adaptations, ed. by Boaz Huss, Marco Pasi, Kocku von Stuckrad, Leiden, Brill, 2010, p. 151-166. Prothero, Stephen, The White Buddhist: The Asian Odyssey of Henry Steel Olcott, Bloomington, Indiana University Press, 1996.

Santucci, James A., "Blavatsky, Helena Petrovna", in Dictionary of Gnosis \& Western Esotericism, ed. by W. Hanegraaff, Leiden, Brill, 2006, p. 177-185.

The Theosophical Movement, 1875-1950, Los Angeles, Cunningham Press, 1951.

The Theosophist: A Monthly Journal Devoted to Oriental Philosophy, Art, Literature and Occultism, conducted by H. P. Blavatsky, Bombay, Theosophical Society, volume 1, 1879-1880.

The Theosophist online, Theosophical University Press Online Edition, $<$ http://www.theosociety.org/pasadena/theosoph/theos-hp.htm>.

THÉvoz, Samuel, Un horizon infini: explorateurs et voyageurs français au Tibet (1846-1912), Paris, Presses universitaires de Paris-Sorbonne, 2010.

VAN Der Veer, Peter, Imperial Encounters: Religion and Modernity in India and Britain, Delhi, Permanent Black, 2001.

VIdal, Denis, "Max Müller and the Theosophists, or the Other Half of Victorian Orientalism ", in Orientalism and Anthropology, from Max Müller to Louis Dumont, ed. by J. Assayag, R. Lardinois, D. Vidal, Pondy Paper in Social Sciences, Institut français de Pondichéry, 1997, p. 13-24.

Vivenānanda, Swami, The Complete Works of Swami Vivekananda, Mayavati, Advaita Ashrama, 8 vols, 1947.

Yadav, K. C., The Autobiography of Dayanand Saraswati, New Delhi, Manohar, 1987. 
